\title{
A matter of responding to stress
}

Some solids are crystals, and others are amorphous. Liquids are, in general, molecularly disordered. Yet the real fundamental difference between liquids and solids isn't structural, but dynamic: liquids can flow and solids cannot. More technically, the difference lies in the response to a shear stress - solids may deform, elastically, but ultimately stand up to the stress. In contrast, liquids cannot, and instead deform continuously.

Even so, this dynamic distinction isn't quite as clear-cut as it appears, and even some of the most familiar substances don't fall into either category. Stack two waffles with some whipped cream between, and the upper waffle will rest stably, the cream acting as a solid, refusing to ooze out the sides. Push down gently, and it still resists, but only to a point. Sufficient downward pressure will overcome the resistance, and the cream now acts like a liquid, flowing out the sides. It's solid, or liquid - all depending on the applied pressure.

Whipped cream belongs to a class of weird hybrid materials that aren't pure solids or liquids. Think of paints, foams, wet cement, gelled crude oil and toothpaste. These so-called yield stress fluids act as firm solids under low stress, yet like sloshing liquids at high stress. Fittingly, many share properties with that most enigmatic of substances, glass, which seems solid enough, yet also shows fluid properties over long periods of time. Understanding such materials is a pressing matter both for industry and basic science, and involves many fundamental physics puzzles (see Daniel Bonn et al., preprint at http://arxiv.org/abs/1502.05281; 2015).

The essential distinguishing property of any such material is a sharp stress threshold for flow. An ordinary Newtonian fluid such as water or oil always flows under a shear stress, no matter how small, and flows faster with increasing stress. The fluid viscosity reflects how the flow rate increases, typically linearly, with more stress, and more rapidly the lower the viscosity. In contrast, a yield stress fluid won't flow at all under small stress, with flow only commencing above a stress threshold, at which point something breaks down. Beyond this point, flow then generally increases with more applied stress, though not necessarily in a linear way.

The key phrase in the above description is "something breaks down". Much of current research focuses on what that something

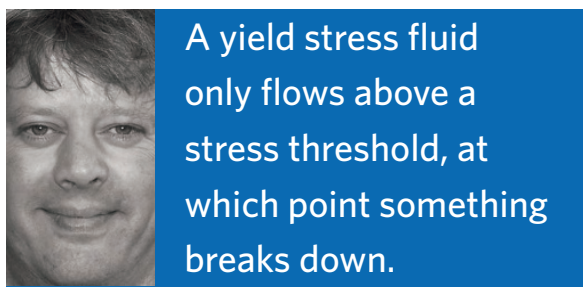

is, how and why it breaks down, and how it reforms when flow eventually stops. While the answer turns out to be different for different materials, a general insight is that yield stress behaviour always depends on the formation under static conditions of physical structures able to resist an applied stress up to a threshold. On the microscopic level, this happens in a number of distinct ways, although the resulting macroscopic behaviour is largely similar in all cases.

One broad class of yield stress fluids arise from molecular liquids - any liquid in which the molecules do not form covalent bonds, but interact only through weak intermolecular forces - if sufficiently cooled. These are ordinary liquids above a glass transition temperature, and flow in response to arbitrarily small shear stresses. Upon cooling to the glassy state, however, molecules find it harder to move and the material develops an effective yield stress threshold. The same holds, as Bonn and colleagues note, in hard-sphere colloidal suspensions, where the transition emerges as the density varies. Experiments find liquid behaviour for low densities, giving way to yield stress behaviour beyond a packing fraction of about 0.58 , where equilibration driven by thermal fluctuations becomes imperceptibly slow, and structures become effectively locked in place. At higher densities, it takes a finite stress to break down the structure and allow flow.

In other systems, especially with larger particles, temperature plays no role; instead, networks built up from physical contacts dominate. For example, foams and systems of large emulsion droplets become solid beyond a critical packing fraction, as the network of repulsive contacts between particles becomes dense enough to support a macroscopic stress. Again, a finite yield stress threshold emerges, with very similar behaviour across a wide class of systems. These materials show a universal power law increase in yield stress close to the critical point.

Finally, in other materials, similar behaviour emerges as a consequence of attractive forces between particles or adhesion. Clay suspensions, for example, are colloidal systems with attractive rather than repulsive interactions between particles. In such systems, as in molecular liquids with attractive forces between molecules, links between particles can, at sufficient density, create a macroscopic stress-supporting structure through a percolation transition.

The common theme, in all cases, is the emergence of a structure able to support a finite stress. The material acts as a solid until the yield stress is overcome, and then flow begins.

When subject to movement, of course, static structures can break down. This is another theme in yield stress materials, and brings a new word into physics: thixotropic. Combining the Greek for touch (thixis) and turning (trope), this refers to a material that turns from one thing into another when touched. Beyond its yield stress threshold, a thixotropic material — such as a suspension of clay particles - becomes a liquid of much lower viscosity, as the structure of its static form dissolves away.

As Bonn and colleagues discuss, thixotropic materials exhibit some spectacularly surprising behaviour. Imagine placing a thick clay suspension on an inclined plane, and then slowly tilting the plane to ever-higher angles. The applied shear stress builds until reaching the threshold, when the material will begin to flow. Naively, one might expect a very small flow rate just above threshold. Yet for a clay suspension, the initial flow, by changing the material structure, causes a pronounced decrease in viscosity and an acceleration of the flow. The result is an abrupt avalanche of material down the plane.

This counterintuitive property can sometimes be dangerous. Prolonged rain can turn the apparently stable soil of a hill or mountain slope thixotropic, so that any small movement can trigger a vast mudslide carrying on much further and more violently than any analysis of the static soil would suggest. In other settings it is merely annoying - as when the ketchup in a bottle stubbornly refuses to budge, despite great effort, until finally, all at once, it cascades out as if it were water.

Solid or liquid? The question is far more subtle than it first appears.

MARK BUCHANAN 